\title{
Drug dosing in neonates
}

\section{Correia}

Department of Anaesthesia, University of the Witwatersrand, South Africa

Corresponding author, email: drmichelle.correia@gmail.com

Keywords: drug dosing, neonates, pharmacokinetics, pharmacodynamics

\section{Introduction}

Neonates have specific physiological differences from older children and adults that influence drug pharmacokinetics (PK) and pharmacodynamics (PD). ${ }^{1-4}$ These differences necessitate adjustments in drug dosing in order to ensure efficacy and avoid toxicity in the perioperative period. Knowledge pertaining to the ontogeny of neonatal organ systems is required to ensure safety and optimal care of these patients.

The myriad of moral and operational concerns pertaining to neonatal research limits our ability to perform prospective trials and gain sufficient data to determine ideal drug dosing in this heterogenous population. Certain advances in PK and PD modelling, especially allometric theories, have improved our recent understanding and highlighted that age, maturation of organ systems and patient size must be taken into account. ${ }^{2-4}$

\section{Definitions ${ }^{3-5}$}

Neonates are defined as children aged from birth to 28 days of life. This group, however, includes a diverse population ranging from extreme prematurity, birth from 22 weeks gestation, to term babies, born after 37 weeks gestation. This term extends to those born prematurely that may be older than 28 days. Accordingly, the post-menstrual age for a newborn, as defined below, can extend from 22 to 50 weeks with corresponding weight ranges from 500 grams to 5 kilograms.

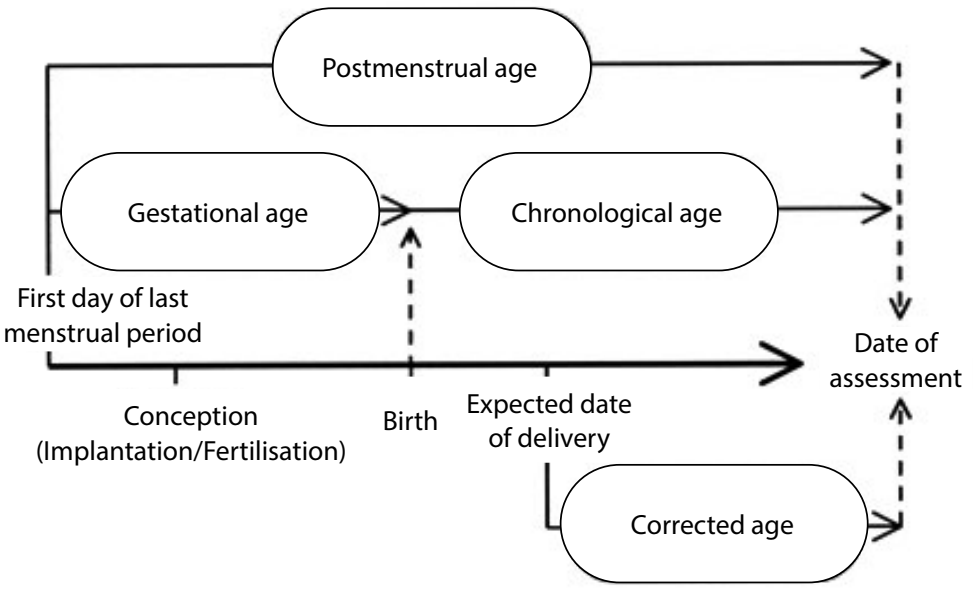

Figure 1: Age terminology during the perinatal period 5
Because age is a key determinant of drug dosing, accurate descriptions are needed to better predict PK and PD parameters. Studies support the use of post-menstrual age as a tenable measure of organ system maturity.

Gestational age is defined as the time from the first day of the last normal menstrual period to the day of delivery. It is expressed in terms of completed weeks.

Post-menstrual age is the gestational age plus the number of weeks since birth. Post-menstrual age is most commonly used for naturally occurring pregnancies as mothers often know the date of their last menstrual period.

Post-conceptual age is used for pregnancies arising from assisted fertility methods as the day of embryo transfer and the day of conception can be accurately assessed. Gestational age can be calculated by adding two weeks to the conceptional age.

Post-natal or chronological age is defined as the time elapsed after birth. It is expressed in days, weeks, months or years.

Corrected age is the chronological age minus the number of weeks born before 40 weeks gestation. This term is used for children who were born premature up to three years of age and is expressed in weeks or months.

As an example using the above definitions a preterm infant born at a gestational age of 31 weeks who has a chronological age of 10 weeks old at the date of assessment would have a corrected post-menstrual age of 41 weeks. This is important to discern as the maturation of organ systems will depend on the corrected post-natal age of the patient. This will determine the anaesthetic technique, including which drug and the doses thereof are selected, as well as the need for special postoperative apnoea monitoring.

\section{Physiological differences in the neonate}

Neonates have particular anatomical and physiological differences that dictate a change in approach. PK and $P D$ variables change rapidly within the first few months of life as organ and enzymatic systems mature. 


\section{Pharmacokinetic differences ${ }^{1-4,6-12}$}

Absorption, volume of distribution ( $\mathrm{Vd})$, metabolism and clearance are all important PK principles that need to be considered. Time-concentration curves are modelled by assessing the $\mathrm{Vd}$, which will determine the loading dose, and clearance, in order to discern a maintenance dose or infusion rate. PK curves allow for safe dosing that ensures clinical efficacy and minimises adverse effects.

\section{Absorption}

The efficacy of a given drug will depend on the rate and extent of absorption from the organ system to which it is administered.

\section{- Enteral route}

- For oral administration, this is dependent on the gastrointestinal tract (GIT) blood flow, $\mathrm{pH}$ and volume of gastric secretions, gastric emptying time and the pharmacological characteristics of the drug itself.

- Enteral absorption is delayed in neonates leading to prolonged time to peak effect and decreased peak concentrations.

- GIT blood flow may be affected by pathological conditions such as sepsis or congenital disease.

- The $\mathrm{pH}$ and volume of gastric contents is variable at birth leading to unpredictable drug absorption characteristics. Gastric content $\mathrm{pH}$ is $6-8$ at birth and decreases to 1.2 on day two of life. An acidic gastric and duodenal environment will allow drugs with a low Pk to have an increased ratio of non-ionised to ionised particles in turn allowing easier passage across lipid membranes and an increased target organ effect.

- Gastric emptying only fully matures at 6-8 months of age owing to age dependent modulation of antral contraction. Disease states such as pyloric stenosis, intestinal atresias or sepsis, and the type of feed containing high calorie and increased long chain free fatty acid densities will further disrupt absorption. Preoperative fasting times need to be adjusted depending on the type of feed the baby is taking.

- Neonatal intestinal motility is decreased due to age dependent variation in intestinal motilin receptor expression. This leads to decreased response to prokinetic agents especially in premature infants.

- Polypharmacy with concurrent administration of other drugs, such as opioids, can slow gastrointestinal motility even further.

- Biliary acid salt production is decreased and negatively influences the solubility and absorptive characteristics of lipophylic drugs.

- Rectal administration of drugs in neonates is associated with variable absorption and efficacy. Bioavailability following repeated doses is affected by decreased gastrointestinal motility and depth of insertion making it an unreliable method of repeat administration.

\section{- Transdermal route}

- Neonates have a higher body surface area (BSA) to weight ratio, an undeveloped control of vasomotor tone, immature stratum corneum and well hydrated epidermis which renders transdermal administration of drugs unpredictable, leading to unintentional toxicity. Fever will cause increased blood supply to the skin and a further increase in absorption.

- The risk of methaemoglobinaemia is higher in neonates because of decreased methaemoglobin reductase activity and the propensity of fetal haemoglobin to become oxidised. Local anaesthetic creams are therefore not repeatedly applied in these patients.

- Increased percutaneous absorption can be further demonstrated by transient hypothyroidism after the cutaneous application of iodine solutions in neonates.

- Inhalational route

- Volatile anaesthetic delivery to the pulmonary vascular bed is dependent on alveolar ventilation, functional residual capacity (FRC), cardiac output and agent solubility.

- Relative to adults, neonates have an increased alveolar ventilation but decreased FRC owing to their increased chest wall compliance. Paired with elevated cardiac output and the greater ratio of blood distributed to vessel rich areas, neonates have a faster equilibration of inspired and alveolar concentrations $\left(\mathrm{F}_{\mathrm{A}}: \mathrm{F}_{\mathrm{i}}\right)$ of anaesthetic agents.

- Tissue: gas solubility of volatiles is approximately $50 \%$ of that in adults. A decreased solubility ratio translates into faster times for partial pressure equilibration of $F_{A}: F_{i}$ and more rapid induction of anaesthesia, especially with the more insoluble agents such as sevoflurane.

- Minimum alveolar concentration (MAC) requirements are decreased by $25 \%$ in neonates compared with infants owing to the immaturity of the central nervous system (CNS) and residual circulating maternal progesterone and $\beta$ endorphins.

- Volatiles depress cardiovascular function, increase respiratory rate, decrease FRC and decrease tidal volumes before suppressing airway reflexes. Decreased tone and collapse of upper airway structures occurs early and supportive measures, such as providing $5-10 \mathrm{CmH}_{2} \mathrm{O}$ positive endexpiratory pressure (PEEP), are usually required to maintain airway patency during inhalational induction.

- Congenital disease states may affect volatile absorption and effect:

- Cyanotic heart lesions associated with right to left shunts and decreased pulmonary blood flow will result in slower inhalational induction of anaesthesia, especially when using the less soluble agents such as sevoflurane and nitrous oxide.

- Left to right shunts usually do not affect the speed of inhalational induction unless there is associated congestive cardiac failure with decreased cardiac output and peripheral perfusion. In this case, although the alveolar 


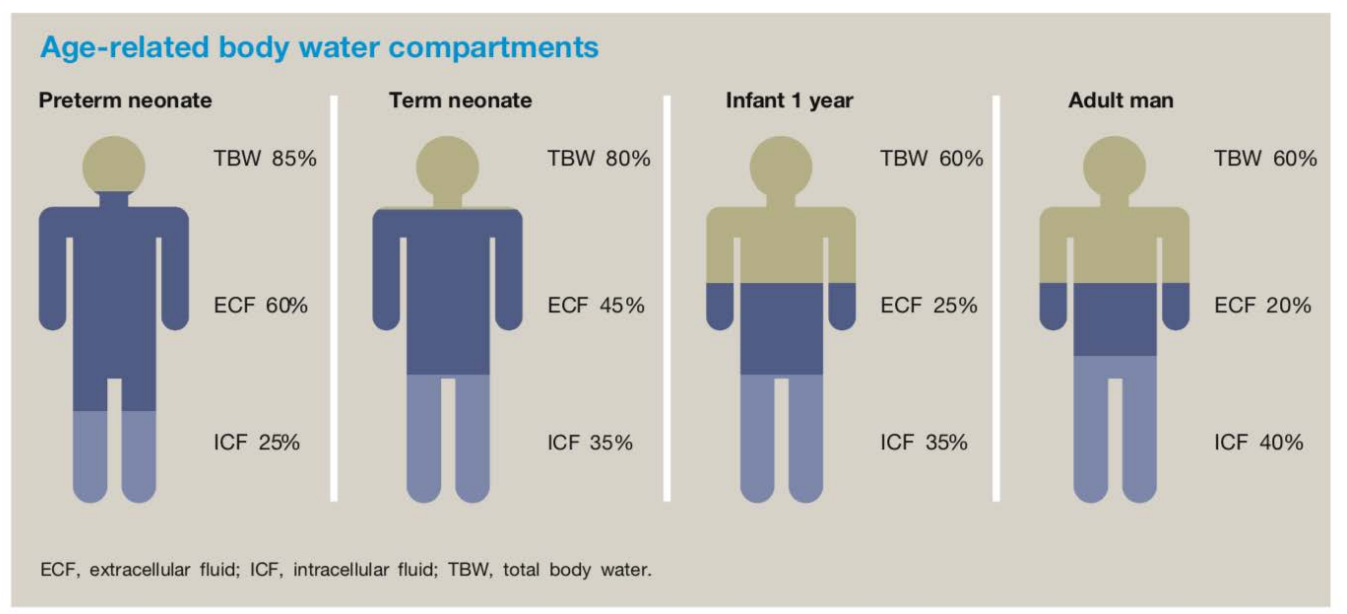

Figure 2: Age related body water compartment composition ${ }^{4}$

partial pressure of the anaesthetic agent will rise quickly, tissue partial pressure will increase more slowly leading to a delayed onset of anaesthesia.

- Intravenous administration (IVI) will result in $100 \%$ bioavailability of the drug administered and is the preferred route for perioperative management.

- Muscle mass, contractility and perfusion are all decreased in neonates leading to slower absorption and insufficient peak plasma concentrations following intramuscular injection.

- Non-traditional routes of administration may be used in neonates because of their specific anatomy and physiology. Interosseous injection into the tibial tuberosity of emergency drugs, using the same dose as for intravenous use, can be lifesaving.

\section{Distribution}

The Vd for any drug is the apparent volume in which the administered dose needs to be dissolved in order to yield a particular plasma concentration. If a drug has a high solubility in water, i.e. is hydrophilic, body composition and total water content will influence the $\mathrm{Vd}$. If the drug has an increased affinity for uptake by tissue, i.e. is highly lipophilic, it will have a large Vd. Decreased cardiac output will change both the $\mathrm{Vd}$ and clearance of drugs administered by affecting tissue perfusion and hepatic blood flow. The degree of protein binding, regional blood flow and specialised physiological barriers all influence the overall distribution of a drug.

\section{- Fluid compartments}

- Body water compartments change dramatically in the first year of life. The highest percentage of total body water is in the premature infant and decreases proportionally with increasing post-natal age. (Figure 2)

- Total body water (TBW) content decreases from $85 \%$ in the premature neonate to $60 \%$ at one year of age.

- Extracellular fluid (ECF) and intracellular fluid (ICF) compartments comprise $60 \%$ and $25 \%$ of TBW respectively in the premature neonate vs $25 \%$ and $35 \%$ in a 1 -year old infant.
- Initial reabsorption of interstitial lung fluid leads to expansion of extracellular fluid volume during the first few days of life. This is followed by a consequent natriuresis and diuresis over the next few weeks.

- This means that the volume of distribution for water soluble drugs (neuromuscular blockers, aminoglycosides) is greatly increased in neonates, especially those who are premature. These drugs distribute rapidly into the ECF compartment after IVI but enter cells more slowly. In order to achieve a sufficient peak plasma concentration of water soluble agents, higher loading doses per kg weight are required.

- Cerebrospinal fluid (CSF) constitutes a higher proportion of total body fluid in neonates. This warrants increasing the dose of local anaesthetic used to provide adequate spinal anaesthesia.

\begin{tabular}{cccc}
\hline Weight & $<\mathbf{5 ~ k g}$ & $\mathbf{5}$ to $\mathbf{1 5} \mathbf{~ k g}$ & $>\mathbf{1 5} \mathbf{~ k g}$ \\
\hline $\begin{array}{c}\text { Isobaric or } \\
\text { hyperbaric }\end{array}$ & $1 \mathrm{mg} / \mathrm{kg}^{-1}$ & $0.4 \mathrm{mg} / \mathrm{kg}^{-1}$ & $0.3 \mathrm{mg} / \mathrm{kg}^{-1}$ \\
bupivacaine $0.5 \%$ & $\left(0.2 \mathrm{ml} / \mathrm{kg}^{-1}\right)$ & $\left(0.08 \mathrm{ml} / \mathrm{kg}^{-1}\right)$ & $\left(0.06 \mathrm{ml} / \mathrm{kg}^{-1}\right)$ \\
\hline
\end{tabular}

Figure 3: Dose of local anaesthetic for spinal anaesthesia ${ }^{6}$

\section{- Tissue compartment composition}

- Per unit of weight, neonates have a reduced proportion of fat and muscle compared with older children and adults. This percentage is smallest in the premature neonate with only $3 \%$ of body weight being contributed by fat. In a term neonate, the percentage of fat increases to around $12 \%$ and is $24 \%$ at five months.

- Pharmacological agents that depend upon redistribution to fat and muscle compartments to decrease their plasma concentration, such as propofol and thiopentone, will have higher plasma and CNS concentrations for sustained periods in these patients due to reduced redistribution.

- Fentanyl and remifentanil exhibit an increased $\mathrm{Vd}$ in neonates. This explains why minimal respiratory depression is seen in term neonates even with the administration of large doses of fentanyl of up to $10 \mu \mathrm{g} / \mathrm{kg}$. These high doses 
are, however, associated with dramatically prolonged effects due to decreased clearance.

\section{- Plasma protein binding}

- Albumin and a-1 acid glycoprotein (AAG) are the two most important plasma proteins to which most drugs bind to be transported in the blood stream. Both exhibit decreased concentrations at birth and reach adult levels at about one year of age.

- The degree of protein binding exhibited by a particular drug will influence its PD parameters as it is the portion of unbound drug that diffuses readily across membranes to reach target receptor sites, produce a pharmacological effect and then be available for metabolism and elimination.

- Neonates have a relative acidosis which can change the ionisation of drugs and influence binding to plasma proteins.

- Acidic drugs, such as diazepam, thiopental and phenytoin bind to albumin. Basic drugs, including local anaesthetic agents, bind to AAG.

- The concentration of both albumin and AAG are decreased in neonates, especially those who are born premature. ${ }^{7}$ This translates into less drug being bound to plasma proteins and an increase in the free drug concentration in plasma. Highly protein bound drugs will have an even greater fraction of free unbound drug in plasma and exert a relatively greater effect.

- Albumin concentrations are lowest in premature infants. Increased levels of free fatty acids and unconjugated bilirubin compete with acidic drugs for albumin binding sites. Prevention of binding or displacement of drugs from protein binding sites can lead to toxicity in jaundiced neonates.

- AAG is an acute phase reactant and concentrations are found to increase in response to surgical stress. ${ }^{7}$ Bupivacaine exhibits > 90\% protein binding to AAG. Increased risk of bupivacaine toxicity in neonates treated with continuous epidural infusion within the first 24 hours post surgery is attributable to decreased clearance and continued elevation of unbound drug fraction in the plasma despite relative increases in protein binding.

- Regional blood flow

- The brain, heart and liver are the first organs to be exposed to pharmacological agents during the initial phase of redistribution.

- There exist maturational changes in relative organ mass and regional blood flow.

- Changes in perinatal circulation involving the ductus arteriosus and venosus as well as the potential return to fetal circulation may influence drug delivery, PK and PD.

- Blood-brain barrier (BBB)

- BBB function and integrity develop gradually during fetal development and are thought to be mature at term gestation.
- Highly selective systems expressed within this specialised endothelial barrier modulate the transport of nutrients and drugs into the CNS.

- Unbound, lipophilic molecules with smaller molecular weight and charge diffuse across the neonatal BBB more easily and achieve equilibration more quickly than in older children and adults. This explains why bupivacaine toxicity is associated with neurological sequelae and seizures rather than cardiac arrhythmias in neonates.

- Premature infants are particularly at risk. The immaturity of the BBB coupled with higher relative brain weight, increased ratio of cerebral blood flow to systemic blood flow and increased unbound drug fraction because of decreased protein binding, all result in increased concentrations of drug in the premature neonatal brain.

- Active transport systems exist to prevent accumulation of certain drugs in the CNS. Animal studies show decreased expression of efflux transporter mechanisms on the BBB of neonatal specimens. The lack of sufficient outward transporters will allow accumulation of certain lipophilic drugs in the CNS and necessitate dose adjustment.

- Fentanyl and morphine are actively pumped out of the brain by an ATP-binding cassette protein, P-glycoprotein. Regulation of onset time as well as the degree and duration of analgesic response to opioids is dependent on P-glycoprotein. Pathology, such as fever, co-administration of other drugs and genetic polymorphisms can all affect the function of this system leading to the highly variable interindividual differences observed in neonates given opioid treatment in the perioperative setting.

\section{Metabolism and clearance}

Pharmacological agents are metabolised and eliminated from the body by three main organ systems: hepato-biliary, renal and pulmonary. All of these systems have a relative amount of immaturity at birth that is proportional to gestational age. It is this ontogeny that must be appreciated in order to prevent potentially fatal dosing errors in individual patients.

\section{- Metabolism}

- Hepato-biliary

- The liver is the principal organ for drug metabolism through enzymatic processes that either render drugs favourable for excretion, convert pro-drugs into more potent compounds or potentially produce toxic metabolites.

- Although neonates are capable of conjugation and glucuronidation at birth, the rate of metabolism is delayed and again, is proportional to post-natal age.

- Microsomal enzyme activity is represented by three distinct levels:

- mature at birth but activity decreases with age

- mature at birth with continued activity into adulthood

- immature at birth, which accounts for most systems 
Table I: Variable maturation of cytochrome P450 enzymes ${ }^{4}$

Examples of variable maturation and expression of different cytochrome P450 enzymes. Enzyme activity does not equate to clearance. Clearance is determined by activity, organ size and blood flow.

\begin{tabular}{|c|c|c|}
\hline Enzyme & Example & Enzyme comments \\
\hline CYP1A2 & Caffeine, theophylline, ropivacaine & Absent in neonate, adult level 4-6 months \\
\hline CYP2C9 & Phenytoin, ketamine, ibuprofen & $\begin{array}{l}\text { Enzyme develops rapidly in the postnatal period; Phenytoin half-life is prolonged at } \\
75 \text { hours in preterm neonates; this decreases to } 20 \text { hours at term and eight hours at two } \\
\text { weeks }\end{array}$ \\
\hline CYP2D6 & Codeine, tramadol, $\beta$-blockers & $\begin{array}{l}\text { Usually present at one week postmenstrual age but only } 20 \% \text { of adult activity at one } \\
\text { month. } \\
\text { Variable because of genetic polymorphism: up to } 47 \% \text { of } 3-12 \text {-year-olds cannot convert } \\
\text { codeine morphine }\end{array}$ \\
\hline CYP3A4 & Midazolam, levobupivavaine & Activity low at birth but increases fivefold over three months of life \\
\hline
\end{tabular}

- Enzymatic processes are divided into phase 1 and phase 2 reactions:

- Phase 1 reactions occur via the cytochrome P450 (CYP) iso-enzyme pathway. These reactions produce small structural changes to the drug molecules that decrease its lipophilicity and promote renal excretion. These reactions include oxidation, reduction and hydrolysis.

- CYP enzymes express variable rates of activity and maturation in neonates as depicted in Table II.

- Phase 2 reactions conjugate the functional group on the parent drug or phase 1 metabolite with a hydrophilic endogenous substrate through processes of glucuronidation, sulfation or acetylation.

- Phase 2 reactions exhibit limited activity during fetal life that extends into the neonatal period.

- Metabolism of parent compounds can transform molecules from a pro-drug into a more pharmacologically active compound. For example, uridine diphosphoglucuronosyltransferase (UGT) 2 B7 conjugates morphine to morphine-6-glucuronide.

- Many iso-forms of the same CYP enzyme can exist and exhibit different age dependent rates of maturation:

- The maturation profile of UGT correlates closely with that of the glomerular filtration rate (GFR) of the kidney. This makes physiological sense as production of water soluble compounds is then coupled with the ability to excrete them from the body.

- These reactions can alternatively produce toxic metabolites: the conversion of halothane to trifluoroacetyl chloride by CYP2E1 is responsible for halothane hepatitis in the liver.

- Immaturity of metabolic processes may have clinical advantages. The absence of CYP1A2 activity required for the metabolism of methylxanthines caffeine and theophylline has been successfully exploited to stimulate breathing and prevent apnoeas, hypoxia and failed extubation in at-risk premature neonates.

- Drug metabolism correlates to body mass in a non-linear fashion. This is described by the term allometry. Adjusting drug doses using direct proportions for weight will often result in under dosing of loading doses owing to the vast differences in body composition. However, decreased clearance will result in prolonged effects and potential toxicity. Dosing intervals and total dose over a 24-hour period are therefore different for neonates, especially those who are premature.

\section{- Clearance}

The rate of enzyme activity does not equate to the rate of drug clearance. Clearance is determined by the rate of enzymatic activity, organ size, regional blood flow to and the relative function and maturity of the organ concerned. Most clearance pathways are immature at birth and reach adult capabilities by two years.

\section{- Renal clearance}

- Renal elimination of drugs and their metabolites is either through glomerular filtration or tubular excretion.

- Functional capabilities of the kidneys, both glomerular and tubular, are immature at birth and directly related to post-natal age. Neonates have decreased ability to handle free water and solute loads which in turn increases the half-life of drugs that rely on renal excretion, necessitating dose adjustments.

- GFR normalised for BSA is decreased until about one year of age.

- Term babies have a rapid and dramatic increase in GFR within the first two weeks of life. Active tubular secretion and reabsorption is only about $30 \%$ of adult values at term, reaching full maturation at about one year of age.

Table II: GFR normalised for BSA according to age 8,11 $^{8}$

\begin{tabular}{lc}
\hline Patient population & GFR $\mathbf{~ m l} \mathbf{m i n} / \mathbf{1 3 3} \mathbf{m}^{\mathbf{2}} \mathbf{B S A}$ \\
\hline Premature babies & 25 \\
Term babies & 35 \\
Two weeks old & 60 \\
Six months old & 80 \\
One year old & Same as adult \\
\hline
\end{tabular}

- Premature infants are particularly at risk because nephrogenesis is only complete at 34 weeks gestation. Dramatically decreased creatinine clearance as well as lack of tubular concentrating and diluting abilities make 
these babies especially high risk for hyponatraemia, hypoglycaemia and acidosis particularly during the perioperative period and in the ICU setting. Adequate exogenous fluid and sodium replacement is mandatory to prevent serious complications.

- Elimination of drugs and their metabolites via active secretion and reabsorption improves with renal maturity and increased function, GFR and renal blood flow.

- Aminoglycosides and cephalosporins are eliminated primarily through the kidney and therefore have prolonged half-life in neonates owing to decreased clearance.

- Extra-hepatic clearance pathways

- Tissue esterases

- Non-specific tissue and erythrocyte esterases are responsible for the breakdown of drugs such as remifentanil and atracurium. Hydrolysis and clearance at this level is increased in younger children which is a reflection of size only as allometry has shown similar levels when scaled to a $70 \mathrm{~kg}$ adult.

\section{- Pulmonary}

- Similar factors that govern agent absorption in the lungs pertain to clearance. Faster equilibration of $F_{A}: F_{i}$ and distribution to muscle and fat leads to a faster washout of volatile agents. Halothane, and to a much lesser extent isoflurane and sevoflurane, undergo some hepatic metabolism which will be delayed owing to organ immaturity.

\section{Pharmacodynamic differences $^{1-4,7-13}$}

PD refers to the effect a drug will have on the body and the processes involved. Neonatal responses to pharmacological agents are affected by the immaturity of target receptors to which the drugs bind, underdeveloped processes to transduce these drug-receptor interactions into intracellular messages and the decreased capability of the immature organ to respond to these messages. The PD response will determine both the therapeutic and adverse effects of the drug given. Co-administration of agents and drug interactions need to be considered in the context of underdeveloped neonatal organ systems.

Because of the difficulty in elucidating clinical endpoints in neonates and correlating this with plasma concentrations, there exists a relative paucity of population PD data. Some measurements of effect, such as neuromuscular transmission stimulation for muscle relaxation intraoperatively, are useful in neonates. Repetitive stimulation is, however, not well tolerated because of decreased acetylcholine (ACh) stores. Other clinical measures of effect, such as assessment of depth of anaesthesia based on EEG, are unreliable without specific neonate-derived algorithms. Although many validated pain assessment tools exist, most are of limited use outside of the acute perioperative setting and in the more premature patients who may not be able to mount a consistent physiological response to pain.

\section{PD characteristics of commonly used anaesthetic drugs in neonates}

- Inhalational agents

- The MAC for anaesthetic volatile agents halothane, isoflurane and desflurane is decreased in premature neonates, increasing with post-natal age and peaking at six months. Immaturity of the CNS and increased regional blood flow may explain an increased drug delivery rate and effect.

- Sevoflurane MAC does not change significantly from neonates through to three months and is actually increased (3.3 vs 2.5).

- Benzodiazepines

- Brain development starts in the third trimester and is completed at five years of age.

- Variability in the number of gamma-aminobutyric acid $\left(G A B A_{A}\right)$ receptors and regulation of chloride transporters in the developing brain change with age leading to unpredictable responses.

- Diazepam exhibits a capacity-limited clearance which is immature in neonates causing a dramatically prolonged elimination half-life. $\mathrm{N}$-desmethyldiazepam is the active metabolite of the parent drug and exhibits a similar potency and half-life. Unpredictable respiratory depressive effects and protracted duration of action make diazepam unsuitable for use in neonates.

- Midazolam clearance is prolonged in neonates compared with older children but its active metabolite has negligible effects, making it more suitable for use in these patients. Its dosing can be titrated to clinical response and the desired level of sedation assessed by the COMFORT score. Hypotension is a common side effect after IVI especially when administered with fentanyl.

- Intravenous anaesthetic agents

- Propofol redistribution and clearance are decreased in the neonatal period leading to prolonged and exaggerated effects. Dose adjustment and titration of effect is required to prevent profound hypotension, especially in premature infants and when administered with opiates.

- Thiopentone dose reduction is required owing to decreased redistribution, decreased protein binding, immature BBB allowing more drug to reach the target organ and increased sensitivity of neonatal receptors.

- Ketamine is a highly lipid soluble and undergoes rapid redistribution. It displays a steep concentration-response curve meaning that small increases in serum concentration will cause a dramatic increase in the level of sedation. Decreased clearance causes a prolonged duration of action.

- Analgesic drugs

- Opiates

- Mu receptor expression and functionality is developmentally regulated in neonates. 
- Increased sensitivity to morphine demonstrated in neonates is related to PK rather than PD differences. Immature $\mathrm{BBB}$, reduced protein binding, decreased metabolism and excretion because of immature hepatic and renal function all contribute to the need for reduced doses.

- Morphine clearance increases dramatically in the neonatal period and reaches $80 \%$ of adult values at six months. Clearance in the critically ill will remain reduced despite age related maturation.

- Multiple factors are important in the clearance of fentanyl, including variable age dependent changes in $\mathrm{Vd}$, hepatic blood flow and function. Metabolism by oxidative $\mathrm{N}$-dealkylation to norfentanyl and hydroxylated fentanyl is dependent on enough free drug reaching the liver. Clearance is drastically reduced in the presence of raised intra-abdominal pressure, as in the case of omphalocele repair, owing to a combination of immature enzyme activity and redistribution of blood flow away from the liver.

- Premature infants are particularly at risk for prolonged respiratory depression from opiates administered in the perioperative period. Controlled ventilation or close monitoring for postoperative apnoea is required if these agents are administered for neonatal surgery.

- The half-life for remifentanil is similar in all age groups. In neonates, the $\mathrm{Vd}$ and clearance are greater than in older children and higher initial infusion rates are required with titration thereafter. Bradycardia and hypotension may be profound especially in the critically ill and premature.

- Paracetamol

- IV paracetamol decreases morphine requirements and is an attractive analgesic alternative to opiates in neonates because of the decreased risk of respiratory depression and exacerbation of apnoeic events.

- The majority of paracetamol when used in therapeutic doses is conjugated via sulfation and glucuronidation in the liver producing water soluble substances which are then eliminated via the kidney. A small amount is oxidised via the CYP2E1 to produce $\mathrm{N}$-acetyl-p-benzoquinone imine (NAPQI). This metabolite is subsequently metabolised by the antioxidant glutathione and cleared.

- NAPQI is a highly reactive electrophilic metabolite that binds hepatocyte proteins and can potentially cause cellular necrosis in high enough concentrations.

- Physiological differences in neonates include the reduced activity of CYP2E1 due to organ immaturity with a theoretical protective effect and decreased production of the toxic NAPQI metabolite. This, however, is offset by decreased glutathione reserves and the decreased ability to regenerate glutathione once depleted. The risk of toxicity is therefore still very real.
- Neonates demonstrate an increased Vd for paracetamol and therefore a loading dose of $20 \mathrm{mg} / \mathrm{kg}$ is recommended. Clearance rates are decreased before 40 weeks post-menstrual age but mature rapidly thereafter reaching $50 \%$ and $90 \%$ of adult values at 52 weeks and one year respectively.

- Neuromuscular blocking agents (NMBAs)

- ACh receptors appear at eight weeks gestation. Primitive motor end plates on one side of the muscle fibres form between 9-16 weeks. The number of nerve terminals decrease from 16-24 weeks changing from a polyneuronal to a mononeuronal system. By 31 weeks, the neuromuscular junction (NMJ) appears mature and continues to grow until the end of the first year of life.

- The subunits on nicotinic ACh receptors in skeletal muscle of the fetus consist of $2 \alpha, 1 \beta, 1 \gamma$ and $1 \delta$ units which differ from the adult form ( $2 a, 1 \beta, 1 \varepsilon$ and $1 \delta$ ). The $\gamma$ subunits usually disappear by 31 weeks gestation. These $\gamma$ subunits can potentially return in pathological states such as severe burns, prolonged inactivity and denervation injuries. There is an associated longer time for channel opening of up to 10 times that of the adult form of the receptor leading to drastically increased efflux of potassium ions and potentially fatal hyperkalaemia.

- Neonates exhibit increased sensitivity to NMBAs owing to the decreased release of $\mathrm{ACh}$ from underdeveloped motor end plates resulting in decreased competition for binding sites in the NMJ.

- The relatively increased ECF compartment leads to increased Vd for NMBAs necessitating a larger initial loading dose for some agents. Suxamethonium requires an initial dose of $3 \mathrm{mg} / \mathrm{kg}$ for intubation because of rapid redistribution to the ECF compartment. Decreased clearance causes an overall increase in the duration of effect.

- NMBAs are quaternary ammonium compounds that mimic the nitrogen radical of $\mathrm{ACh}$. There is an increased risk of anaphylaxis associated with suxamethonium, rocuronium and atracurium.

- Local anaesthetic (LA) agents

- Relative deficiency of myelination, due to prematurity or hypoxia induced demyelination injury, altered conduction involving immature nodes of Ranvier and differences in the length of nerve exposed lead to a block of shorter duration and less dermatomal coverage when amide LA agents are administered for spinal anaesthesia in neonates. This, coupled with relatively higher CSF volume:TBW content, necessitates a larger weight-scaled dose.

- Larger Vd reduces peak concentrations after single dosing but decreased metabolism and clearance increases the risk of accumulation with continuous infusions.

- Protein binding to AAG and clearance of bupivacaine are drastically reduced in neonates. Coupled with increased vulnerability of the developing brain, these patients are at 
increased risk of seizures unless infusion rates of epidurals for postoperative pain relief are decreased appropriately. Safe continuous infusion rates of $0.2-0.25 \mathrm{mg} / \mathrm{kg} / \mathrm{hour}$ are suggested.

- Decreased metabolism and clearance of ropivacaine (CYP1A2) and levobupivacaine (CYP3A4) dictates similar considerations for these drugs.

\section{- Inotropes}

- Calcium stores in the endoplasmic reticulum of the neonatal myocardium are reduced. Exogenous calcium has a relatively greater impact on contractility in these patients than in older children and adults. Conversely, calcium channel blocking agents may cause life threatening bradycardia and hypotension.

- Endogenous catecholamine release and responses to vasoactive drugs are age dependent and vary based on the ontogeny of myocardial structure, cardiac innervation and adrenergic receptor function.

- The immature myocardium contains less contractile elements and has a reduced capacity to increase contractility or improve diastole, resulting in poor responses to afterload and preload manipulation.

- Dopaminergic receptors in the pulmonary circulation are involved in pulmonary vasoconstriction. Systemic vasoconstriction is however greater than that in the pulmonary bed and this differential response supports the use of exogenous dopamine in neonates with pulmonary hypertension post cardiac surgery.

- Neonates have an underdeveloped sympathetic nervous system and reduced stores of noradrenaline. $\beta$ receptor maturation lags behind that of a receptors during the development of the adrenergic system. a-agonists have increased effects at lower doses compared with $\beta$-agonists because of this difference in maturation.

\section{Toxicity in neonates}

Certain pharmacological agents used for anaesthesia in neonates have been shown to cause toxicity unique to this population.

\section{Developmental neurotoxicity ${ }^{1,9,14}$}

- Several animal studies have convincingly shown accelerated neuronal apoptosis and long term behavioural changes related to exposure to anaesthesia during the neonatal period. Although there exists a deficiency of prospective data in humans, retrospective reviews demonstrate temporary neurological sequelae after prolonged exposure to certain anaesthetic agents.

- The administration of volatile and IV agents, especially isoflurane and ketamine, are associated with dose dependent and length of exposure, i.e. time dependent, neurodegenerative effects.

- In adults and older children, $\mathrm{GABA}_{\mathrm{A}}$ receptor activation leads to influx of chloride ions, hyperpolarisation of membranes and a decrease in neuronal activity. This may be neuroprotective in times of hypoxia or ischaemia.

- Rapid, extensive and ongoing synaptogenesis is the hallmark of the developing brain.

- During periods of synaptogenesis, intracellular concentrations of chloride are already elevated. Activation of $\mathrm{GABA}_{A}$ receptors and opening of channels in this instance, will cause an efflux of chloride down its concentration gradient and depolarisation of neurones. Membrane depolarisation then causes an influx of calcium.

- Increased intracellular calcium concentrations are associated with apoptotic neurodegeneration and neuronal injury that has been linked to memory and learning deficits later in life.

- This mechanism is known as the excito-toxic action of $\mathrm{GABA}_{\mathrm{A}}$ receptors and is triggered with the administration of benzodiazepines and other anaesthetic agents.

- NMDA receptor antagonists such as ketamine are thought to promote excito-toxicity via a mechanism of compensatory increases in the number of receptors expressed after sustained blockade. After clearance of the drug, the exaggerated influx of calcium can lead to apoptosis and cell death.

- The developing brain produces immeasurably more neurones than those that are needed and survive into adulthood. Active processes involving apoptosis and synaptic pruning are crucial to overall plasticity and ensuring normal development. Neurotrophin signalling mechanisms are instrumental in this process. Isoflurane is thought to affect the balance of neurotrophic factors leading to neuronal injury.

- The risk of neurotoxicity must be weighed against the risk of inadequate anaesthesia and analgesia. Deficient anaesthesia and untreated pain cause prolonged physiological and behavioural changes in and of themselves.

- All elective surgery should be postponed until 60 weeks postmenstrual age.

- There is no data supporting the use of one agent over another, although ketamine, midazolam and isoflurane have been specifically indicated.

- Single exposure to general anaesthesia for less than one hour has not been associated with neurotoxicity even in patients who are less than 60 weeks post-menstrual age.

\section{Oxygen toxicity ${ }^{15,16}$}

- The association between supplemental oxygen therapy and toxicity in neonates was first discovered in the 1950s when retinopathy was diagnosed in premature infants breathing high concentrations of inspired oxygen.

- Oxygen toxicity occurs because of the formation of reactive oxygen species (ROS). In the first few minutes after birth, the blood oxygen content in the newborn increases dramatically as oxygen availability increases. This causes a burst generation of ROS.

- There are no specific endogenous scavengers for these radicals. Once released, ROS react with a number of intracellular and 
cell membrane molecules causing structural and functional damage to these structures.

- Oxidative stress is the term used to describe the balance between oxidants, toxic ROS that can cause cell damage, and antioxidants. Premature infants are particularly at risk because they have immature and limited antioxidant defence mechanisms.

- Chronic oxygen exposure to the lungs limits lung growth leading to broncho-pulmonary dysplasia, damages pulmonary epithelium, inactivates surfactant causing atelectasis, interstitial thickening, fibrosis and ultimately chronic lung disease.

- Retinopathy of prematurity is caused by stimulation of vascular endothelial growth factor and neovascularisation of the retina leading to fibrosis and detachment. Both hyperoxia and hypoxia have been linked to retinopathy suggesting that avoiding fluctuations in saturations is of utmost importance.

- Neurologically, oxidative stress has been linked to intraventricular haemorrhage, the highest risk for development being in the first five days after birth with the transition to extrauterine life; and periventricular leukomalacia which is thought to occur because of oxidative damage to oligodendrocytes in the white matter.

- As with any other drug, oxygen has a risk:benefit ratio and must be administrated in appropriate doses. Monitoring saturations and targeting appropriate levels is mandatory in the management of neonates.

\section{Conclusions}

Neonates are not a homogenous group of patients. The population is represented by a wide cohort exhibiting differing maturation of organ systems, weight and age ranges and pathologies. These factors contribute to the degree of interindividual variability observed in both PK and PD parameters in these patients. Size, age and organ maturation are the main covariates that effect PK criteria. The majority of PK processes are immature at birth and mature at some stage during the first two years of life. Post-menstrual age is the most useful measure of PK organ system maturity. PD differences include changes in $M A C$ values for volatiles agents, paradoxical $G_{A B A_{A}}$ receptor effects and altered responses to NMBAs and inotropic drugs. Drug dosing in these patients requires careful assessment and individualisation. Possible toxicity with anaesthetic agents and oxygen must be remembered and the conduct of anaesthesia for these patients carefully considered.

\section{Conflict of interest}

The author declares no conflict of interest.

\section{Funding source}

None.

\section{ORCID}

\section{Correia (iD https://orcid.org/0000-0001-7171-0040}

\section{References}

1. Maheshwari M, Sanwatsarkar S, Katakwar M. Pharmacology related to paediatric anaesthesia. Indian J Anaesth. 2019;63:698. https://doi.org/10.4103/ija. IJA_487_19.

2. Anderson BJ, Holford NHG. Understanding dosing: children are small adults neonates are immature children. Arch Dis Child. 2013;98:737-44. https://doi. org/10.1136/archdischild-2013-303720.

3. Anderson BJ, Allegaert K. The pharmacology of anaesthetics in the neonate. Best Pract Res Clin Anaesthesiol. 2010;24:419-31. https://doi.org/10.1016/j. bpa.2010.02.019.

4. Anderson BJ. Neonatal pharmacology. Anaesth Intensive Care Med. 2017;18:68 74. https://doi.org/10.1016/j.mpaic.2016.11.007.

5. Engle WA, Blackmon LR, Batton DG, et al; Committee on Fetus and Newborn. Age terminology during the perinatal period. Pediatrics. 2004;114:1362-4. https://doi. org/10.1542/peds.2004-1915.

6. Troncin R, Dadure C. Paediatric spinal anaesthesia. Management. 2009;4:8.

7. Anell-Olofsson $M$, Ahmadi $S$, Lonnqvist PA, et al. Plasma concentrations of alpha-1-acid glycoprotein in preterm and term newborns: influence of mode of delivery and implications for plasma protein binding of local anaesthetics. $\mathrm{Br}$ Anaesth. 2018;121:427-31. https://doi.org/10.1016/j.bja.2018.01.034.

8. Martin LD. The basic principles of anesthesia for the neonate. Revista Colombiana de Anestesiologia. 2017;45:54-61. https://doi.org/10.1016/j. rca.2016.07.006.

9. Zisovska E. Anaesthetics safe in neonates: proposal for update of Section 1 ANAESTHETICS included in the 3rd WHO Model List of Essential Medicines for Children. 2011. Available from: http://158.232.12.119/selection_medicines/ committees/expert/19/applications/Anaesthesianeonates_1_C_R.pdf.

10. Martin L, Jimenez N, Lynn AM. Developmental pharmacokinetics and pharmacodynamics of parenteral opioids and nonsteroidal anti-inflammatory drugs in neonates and infants. Revista Colombiana de Anestesiologia. 2017;45:72-9. https://doi.org/10.1016/j.rcae.2016.11.011.

11. Haidon $J$, Cunliffe M. Analgesia for neonates. Continuing Education in Anaesthesia Crit Care Pain. 2010;10:123-7. https://doi.org/10.1093/bjaceaccp/ mkq016.

12. Ku LC, Smith PB. Dosing in neonates: special considerations in physiology and trial design. Pediatr Res. 2015;77:2-9. https://doi.org/10.1038/pr.2014.143.

13. Nasr VG, Davis JM. Anesthetic use in newborn infants: the urgent need for rigorous evaluation. Pediatr Res. 2015;78:2-6. https://doi.org/10.1038/pr.2015.58.

14. Perouansky $M$, Hemmings $H C$, Riou B. Neurotoxicity of general anesthetics: cause for concern? Anesthesiology. 2009;111:1365. https://doi.org/10.1097/ ALN.0b013e3181bf1d61.

15. Kayton A, Timoney P, Vargo L, Perez JA. A review of oxygen physiology and appropriate management of oxygen levels in premature neonates. Adv Neonatal Care. 2018;18:98. https://doi.org/10.1097/ANC.0000000000000434.

16. Perrone S, Bracciali C, Di Virgilio N, Buonocore G. Oxygen use in neonatal care: a two-edged sword. Front Pediatr. 2017;4:143. https://doi.org/10.3389/ fped.2016.00143. 